\title{
Parametric microstructure modeling of compressed cathode materials for Li-ion batteries
}

\author{
Benedikt Priflinga,*, Daniel Westhoffa ${ }^{\mathrm{a}}$, Denny Schmidt ${ }^{\mathrm{b}}$, Henning Markötter ${ }^{\mathrm{c}}$, Ingo Manke ${ }^{\mathrm{c}}$, \\ Volker Knoblauch ${ }^{\mathrm{d}}$, Volker Schmidt ${ }^{\mathrm{a}}$ \\ ${ }^{a}$ Institute of Stochastics, Ulm University, 89069 Ulm, Germany \\ ${ }^{b}$ Robert Bosch Battery Systems GmbH, 70469 Stuttgart, Germany \\ ${ }^{c}$ Institute of Applied Materials, Helmholtz-Zentrum Berlin, 14109 Berlin, Germany \\ ${ }^{d}$ Materials Research Institute, Aalen University, 73430 Aalen, Germany
}

\begin{abstract}
The microstructure of electrodes significantly influences the electrochemical performance of lithium-ion batteries. Thus, a deeper understanding of the electrode microstructure provides valuable information for the design of optimized electrode morphologies. One promising approach is called virtual materials testing, where stochastic microstructure models are used for generating a large number of virtual, but realistic morphologies, which serve as input for numerical transport simulations. Doing so, relationships between the microstructure and functional properties of the electrodes can be investigated. In the present paper, we utilize a parametric stochastic microstructure model, which is calibrated to tomographic image data of eight differently manufactured cathodes, where the compaction load has been varied. Since one and the same model type is used for all compaction loads, we can predict the model parameters for an arbitrary compaction load. This allows us to perform predictive simulations, i.e., we are able to generate virtual microstructures that correspond to a compaction load which has not been observed experimentally. The goodness of fit of the microstructure model is validated by comparing phase-based as well as particle-based characteristics of model realizations and tomographic image data. In addition, the suitability of the stochastic microstructure model for predictive simulations is pointed out by cross-validation.
\end{abstract}

Keywords: stochastic modeling, lithium-ion batteries, cathodes, microstructure, prediction, compaction

\section{Introduction}

In recent years, lithium-ion batteries have proven to be one of the major technologies for storing energy since they combine a low self-discharge rate with an outstanding energy density. Lithiumion batteries are, among others, used in portable electronic devices such as laptops and smart 5 phones as well as in large-scale applications like electric vehicles [1].

${ }^{*}$ Corresponding author. Email: benedikt.prifling@uni-ulm.de. Phone: +49 73150 - 23555. Fax: +49 73150 23649. 
It is well known that the microstructure of anodes and cathodes strongly influences their electrochemical properties, see [2], [3] and [4] for the effect of particle size and shape, [5] for the importance of cracks in active material particles and [6] for the relevancy of spatially localized features. Therefore, the development of optimized electrode morphologies by systematically structuring the

\section{clectrodes is an inportant task in materials science, see [7] and [8]. The cathode manufacturing} process, which includes mixing, coating, drying and calendering, is of great interest since it significantly influences the resulting electrode microstructure. In [9], [10], [11] and [12], the strong influence of the calendering process on the electrochemical performance has been investigated. In [13] it has been shown that the calendering process interacts with other processing steps such as 10 mixing. In addition, the amount and distribution of carbon black and binder is another important influencing factor [14].

In [15], an extensive statistical analysis of three-dimensional (3D) tomographic image data has been performed in order to quantify the changes of microstructure that arise during the calendering process of a cathode. In the present paper, we describe how a parametric stochastic microstructure model is fitted to the eight differently compacted cathodes considered in [15]. Note that the calendering process itself is not modeled. Instead, model parameters are chosen in such a way that the presented microstructure model generates virtual cathode microstructures that exhibit similar structural properties compared to tomographic image data of differently compacted samples. Stochastic microstructure modeling is an important part of an approach called virtual materials testing. The basic idea is to calibrate a parametric stochastic microstructure model to tomographic image data by means of tools from stochastic geometry, see [16]. By generating multiple model realizations as well as by varying the model parameters, a broad range of virtual, but realistic morphologies can be generated. Based on a large number of virtual microstructures, numerical simulations can be used to compute functional characteristics, which allows for investigating microstructure-property relationships. This valuable information facilitates the design of optimized microstructures of materials with preferable functional properties. For examples, where virtual materials testing has been performed, we refer to [17] for a study of open-cell foams as well as to $[18,19]$, where the effect of morphological characteristics on effective conductivity and permeability has been investigated.

The rest of this paper is organized as follows. In Section 2, the tomographic image data will be shortly described. Afterwards, the basic ideas of the stochastic microstructure model are explained. In Section 4, the calibration of the stochastic microstructure model for each compaction load is described. Section 4 deals with the quantitative validation of the parametric model by comparing several characteristics describing the cathode morphology. Since the stochastic model is parametric, we are finally able to predict all model parameters and hence, the complete morphology for arbitrary compaction loads, see Section 5. In addition, we perform a leave-one-out cross-validation in order to quantify the goodness of fit. Section 6 concludes the paper with a short summary and discussion of the main results.

\section{Data description}

At this point, we shortly summarize the image preprocessing results obtained in [15], which will be the basis for the subsequent stochastic modeling considered in the present paper. In [15], 
the microstructure of eight cathodes, each with the same loading level of $2.46 \mathrm{mAhcm}^{-2}$, has been considered, where for each cathode a different compaction load has been applied during the densification process. Further details regarding the uniaxial compaction procedure can be found in [20]. The uncompacted sample ( $0 \mathrm{MPa}$ ) exhibits a porosity of $49.8 \%$, whereas the highest compaction

\section{of the (1000} of the cathodes as well as their electrochemical performance can be found in [20]. Tomographic $3 \mathrm{D}$ image data has been obtained via synchrotron tomography, whereby the resulting 3D grayscale images have been preprocessed in two major steps. In a first step, a phase-based segmentation has been performed, which resulted in two phases, i.e., the active material phase $\left(\mathrm{LiNi}_{1 / 3} \mathrm{Co}_{1 / 3} \mathrm{Mn}_{1 / 3} \mathrm{O}_{2}\right.$, briefly called NCM in the following) and a phase consisting of binder as well as conductive additives and the pore space. Note that a subdivision of the second phase into pore space and a combination of binder and conductive additives has been modeled based on the composition of the active mass but without direct reference to tomographic image data, since binder and additives are not visible in the synchrotron images. Afterwards, a particle-based segmentation has been performed using a marker-based watershed algorithm with extended regional minima, which has been complemented with a post-processing step. Based on the preprocessed 3D image data, a large variety of microstructural characteristics has been computed. Later on, these characteristics will be used for quantifying the goodness of fit of the calibrated stochastic microstructure model. Note that the subsequent sections of the present paper mainly focus on the stochastic modeling of the active particle system, i.e., the system consisting of NCM-particles.

\section{Model description}

In this paper, we make use of the stochastic microstructure modeling interface described in [21]. The main ideas are summarized in the following.

In the first step, a non-overlapping sphere packing is generated in a cubic sampling window $W \subset \mathbb{R}^{3}$. The spheres act as placeholders for the active particles, i.e., they model their locations and approximate sizes. To obtain such a system of non-overlapping spheres, we start with a system of overlapping spheres, i.e., we draw the radii from a particle size distribution $\mathcal{R}$ and place the midpoints uniformly distributed in $W$ until the sum of the volumes of all spheres divided by the volume of the sampling window reaches a certain (predefined) fraction $\xi \in(0,1)$. Note that the particle size is expressed by using volume-equivalent radii. Afterwards, a force-biased collective rearrangement algorithm is applied $[22,23]$. This leads to a non-overlapping sphere packing as long as $\xi$ is not too large, since the algorithm described above is only able to achieve packing densities up to $\approx 65 \%$. For $\xi>60 \%$ we make use of a so-called core-shell ratio $\gamma \in(0,1)$ to generate systems of slightly overlapping spheres as described in [21]. The result of the first modeling step is a marked point pattern $\Xi=\left\{\left(S_{i}, R_{i}\right): i=1, \ldots, N\right\}$, where $N$ is the random number of spheres and $S_{i}$ and $R_{i}$ are the center and radius of the $i$-th sphere, respectively.

The second step is to define a suitable connectivity graph $G=(V, E)$, where the set of vertices 40 $V=\left\{S_{i}: i=1, \ldots, N\right\}$ is given by the sphere midpoints obtained by the force-biased algorithm in the first modeling step. The edge set $E \subset V \times V$ is a subset of pairs of points. If there is an edge between two points $S_{i}$ and $S_{j}$, the particles, which will be created in the third step, have to 
touch at a certain matching point. If a vertex is not connected with other vertices, there are no constraints that have to be fulfilled during the creation of the corresponding particle. Thus, the creation of particles is conditioned on $G$, which allows to control the connectivity of the particle system. In particular, the number of particles always equals the number of spheres.

Finally, at every sphere center $S_{i}$ an active particle is generated using spherical harmonics, see [24] for technical details. This is a reasonable approach since the nearly spherical NCM-particles are star-shaped, which is the only assumption for the creation of complex shaped particles via spherical harmonics. The mean volume of the particles is approximately given by the volume of the underlying spheres. In addition, the generation of a single particle is conditioned on the connectivity graph $G$. If there is an edge in the edge set $E$ connecting two points $S_{1}, S_{2} \in V$, then the corresponding spherical harmonics particles have to touch each other at a certain matching point $m \in \mathbb{R}^{3}$. In our case, the matching point is determined according to the following rule: Let $\left(S_{i}, R_{i}\right)$ and $\left(S_{j}, R_{j}\right)$ denote the underlying spheres. The resulting matching point is given by

$$
m=S_{i}+\frac{\left(R_{i}+0.5 \cdot\left(\left\|S_{i}-S_{j}\right\|-R_{i}-R_{j}\right)\right)}{\left\|S_{i}-S_{j}\right\|} \cdot\left(S_{j}-S_{i}\right),
$$
voxels in the $x y$-plane $(219 \mu \mathrm{m} \times 219 \mu \mathrm{m})$, whereas the number of slices in $z$-direction depends on 
Table 1: Experimentally determined cathode thickness and the corresponding size of the sampling window in $z$-direction for each compaction load.

\begin{tabular}{lcccccccc}
\hline Compaction load $(\mathrm{MPa})$ & 0 & 100 & 200 & 300 & 400 & 500 & 750 & 1000 \\
\hline Cathode thickness $(\mu \mathrm{m})$ & 79.8 & 61.0 & 57.2 & 53.7 & 51.9 & 51.0 & 49.8 & 49.1 \\
Number of voxels in $z$-direction ( ) & 182 & 139 & 131 & 123 & 118 & 116 & 114 & 112 \\
\hline
\end{tabular}

the compaction load, since the mean cathode thickness significantly decreases with increasing compaction load [15]. More precisely, we choose the number of slices in $z$-direction in such a way that the size of the sampling window in $z$-direction is equal to the experimentally determined cathode thickness, see Table 1.

\subsection{Volume fraction}

The volume fractions of NCM-particles have been experimentally determined in [20] for all compaction loads. They are listed in Table 2. Hence, the model parameter $\xi$ is known for all compaction loads. In addition, we have to use the core-shell ratio $\gamma$ for all compaction loads greater than or equal to $200 \mathrm{MPa}$, since these compaction loads exhibit volume fractions of the active material larger than $60 \%$. This quantity is given by $\gamma=\sqrt[3]{\frac{0.6}{\xi}}$.

Table 2: Experimentally determined volume fraction of NCM-particles, mean coordination number of NCM-particles computed from tomographic image data, distance threshold $d$ as well as correction factor $\rho$ as a function of the compaction load.

\begin{tabular}{lcccccccc}
\hline Compaction load $(\mathrm{MPa})$ & 0 & 100 & 200 & 300 & 400 & 500 & 750 & 1000 \\
\hline Volume fraction (\%) & 41.6 & 54.2 & 58.1 & 61.9 & 63.9 & 65.1 & 66.7 & 67.7 \\
Mean coordination number () & 3.50 & 5.91 & 6.67 & 7.51 & 7.66 & 7.92 & 7.99 & 8.42 \\
Distance threshold $d(\mu \mathrm{m})$ & 1.19 & 1.41 & 1.55 & 1.73 & 1.91 & 2.05 & 1.96 & 2.32 \\
Correction factor $\rho()$ & 1.004 & 1.006 & 1.008 & 1.010 & 1.011 & 1.011 & 1.013 & 1.012 \\
\hline
\end{tabular}

\subsection{Particle size distribution}

In [15] it has been shown that the compaction load has nearly no influence on the particle size distribution and particle cracking is neglected. Hence, when modeling the structures, it is reasonable to use one and the same parametric particle size distribution for each compaction load. To do so, we consider the volume-equivalent radii of all observed particles among the entire series of samples, i.e., we do not distinguish between different compaction loads. The histogram of all particle radii, shown in Figure 1, indicates that it is reasonable to introduce a lower bound $s>0$ for the particle radii. In addition, it turns out that the shape of the particle size distribution can be modeled by a Birnbaum-Saunders distribution. More precisely, let $T$ denote a random variable which follows the Birnbaum-Saunders distribution with shape parameter $\gamma>0$ and scale parameter $\alpha>0$, see [25]. The shifting parameter $s$ is chosen in such a way that the log-likelihood function is maximized. For this purpose, we choose 50 equally spaced points $s_{1}, \ldots, s_{50}$ between 0 and $0.725 \mu \mathrm{m}$, 


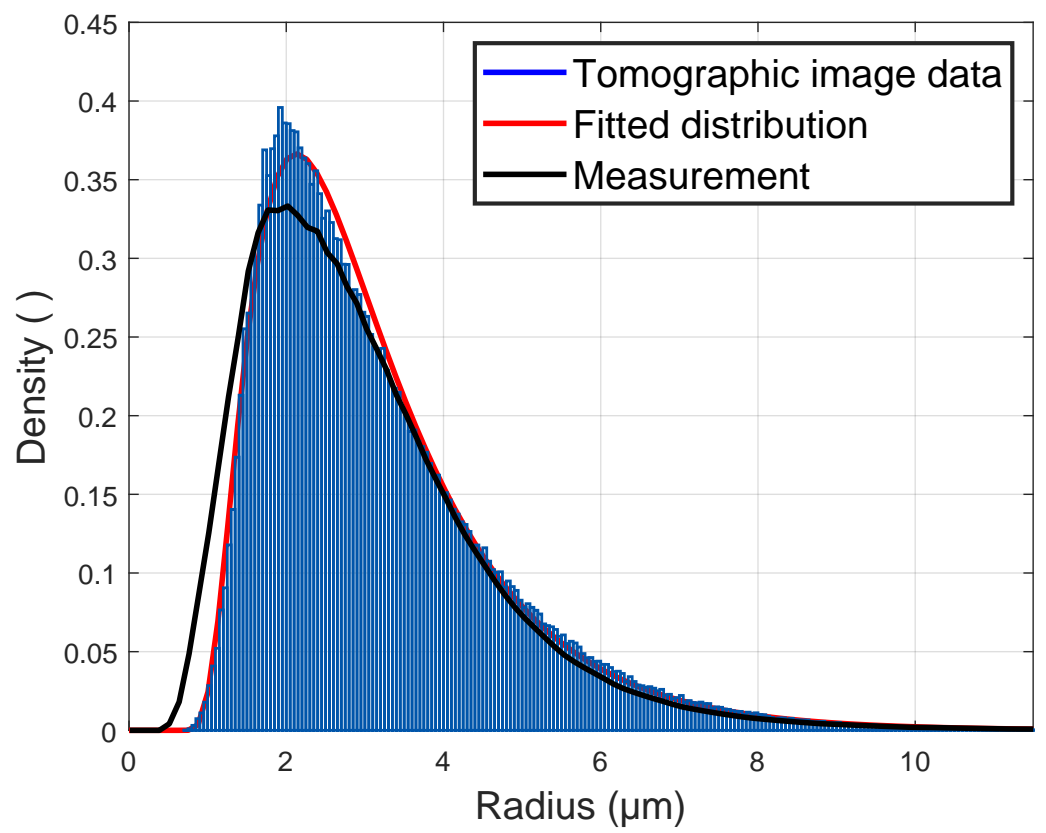

Figure 1: Histogram of observed particle sizes (blue) together with the fitted parametric distribution (red).

where the upper bound of the interval corresponds to the minimal observed radius. For each point $s_{i}$, we consider the particle sizes shifted by $s_{i}$ to the left and fit a Birnbaum-Saunders distribution using the maximum likelihood estimator implemented in MATLAB and calculate the resulting log-likelihood. This procedure leads to $\hat{s}=0.503 \mu \mathrm{m}, \hat{\gamma}=0.5666$ and $\hat{\alpha}=2.2837$. Furthermore, it is meaningful to truncate the distribution of $s+T$ at a certain level $t>0$ in order to eliminate the unlikely case that extremely large particles occur. In our case, we choose $t=11 \mu \mathrm{m}$, which is in good accordance with Figure 1. The black curve in Figure 1 is computed based on experimental measurements of the particle size of active material particles using laser diffraction analysis (Mie theory). Due to the high coincidence between the black curve and the histogram in Figure 1, it can be concluded that the particle-based segmentation of tomographic image data is reasonable.

\subsection{Connectivity graph}

In order to define a suitable connectivity graph $G$, we first have a closer look at the connectivity of NCM-particles in the preprocessed tomographic image data. For each NCM-particle, we are able to determine the number of directly connected neighboring particles (called the coordination number) using the particle-based segmentation considered in [15]. The mean coordination number for each compaction load can be found in Table 2, which exhibits a strictly monotonous behavior as one would expect.

In order to reflect the fact that the mean coordination number increases with increasing compaction load, we define the connectivity graph in dependence of this quantity. More precisely, the connectivity graph $G_{d}=\left(V, E_{d}\right)$ depends on a distance threshold $d>0$ where the edge set is given by

$$
E_{d}=\left\{\left(S_{i}, S_{j}\right):\left\|S_{i}-S_{j}\right\|-R_{i}-R_{j} \leq d\right\}
$$


The parameter $d$ depends on the compaction load, where the values of $d$ are chosen in such a way that the mean coordination numbers of real and simulated electrode microstructures nicely match. For this purpose, the bisection method is used, where we generate ten model realizations in the sampling window $W=[0,250]^{3}$ in each step of the bisection method [26]. The corresponding

5 values of $d$ are given in Table 2 .

\subsection{Particle shapes}

This section deals with the creation of NCM-particles using spherical harmonics. For this purpose, the angular power spectrum $A:[0, \infty) \rightarrow[0, \infty)$ is estimated for each compaction load using the particle-based segmentation considered in [15], which directly influences the shape of the generated particles. The results are visualized in Figure 2. As it can be clearly seen, the compaction load has almost no influence on the angular power spectrum. Hence, we calculate the pointwise averaged angular power spectrum $A_{\text {average }}:[0, \infty) \rightarrow[0, \infty)$ (black dots in Figure 2 ).

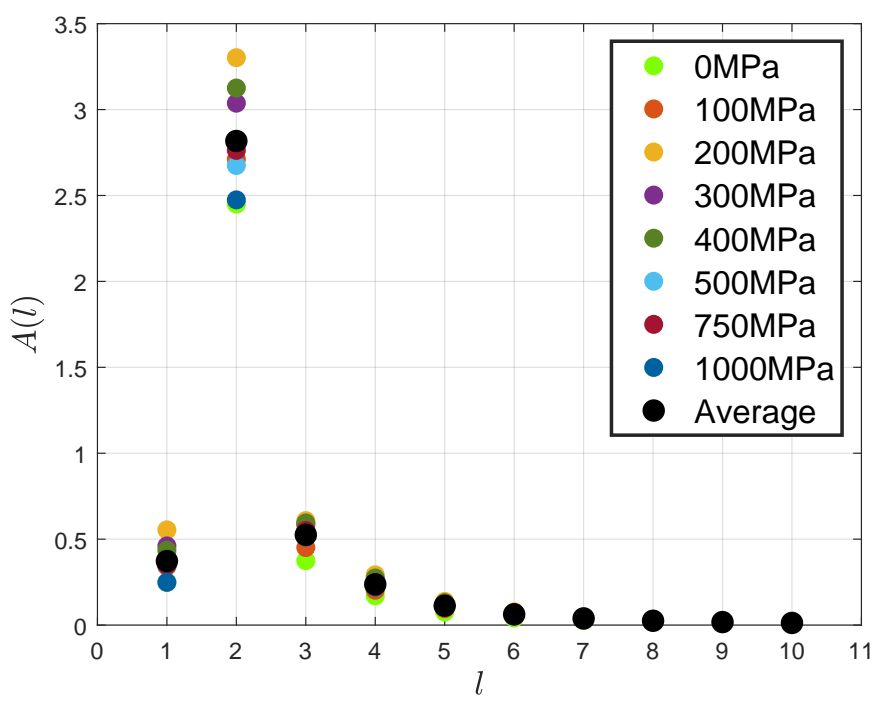

Figure 2: The estimated angular power spectrum for each compaction load, together with the pointwise averaged angular power spectrum (black).

In addition, the series expansion parameter $L=7$ has been used for all compaction loads since this choice of $L$ allows us to capture the shape of active material particles observed in tomographic image data. As it has been described in [21] and [27], it is possible that during the generation of a spherical harmonics particle the radius function $\psi:[0, \pi] \times[0,2 \pi)$ takes negative values. If this is the case, we reject the current radius function and draw a new one until the radius function takes positive values only. This acceptance-rejection step is performed up to $\eta$ times. If after $\eta$ trials, the radius function still has negative values, we keep the underlying sphere as a model for the corresponding particle. Note that this usually happens for rather small particles, which will only consist of a few voxels in the discretized image. In the following, we use $\eta=50$.

\subsection{Post-processing}

Generating virtual microstructures according to the procedure described above leads to realistic cathode morphologies, which reflect the key features of the tomographic image data. However, 
the underlying connectivity graph only ensures that two particles touch at exactly one location, which does not reflect the appearance of tomographic image data. A post-processing allows us to enlarge the interface area between two particles. Moreover, this post-processing lowers the specific surface area of the particle phase, which otherwise would be significantly too large. The 5 post-processing is performed as follows. A 3D Gaussian filter with the same standard deviation $\sigma_{x}=\sigma_{y}=\sigma_{z}=0.438 \mu \mathrm{m}$ in all directions is applied to the binary image, see [28] for details. This leads to a grayscale image. In order to again obtain a binary image, we apply a global threshold, which is calculated with Otsu's method [29]. Note that this procedure leads to a similar effect as a morphological closing. However, this approach described above has been chosen since the 10 shape of the NCM-particles is preserved to a large extent. Figure 3 visualizes the effects of this post-processing step.
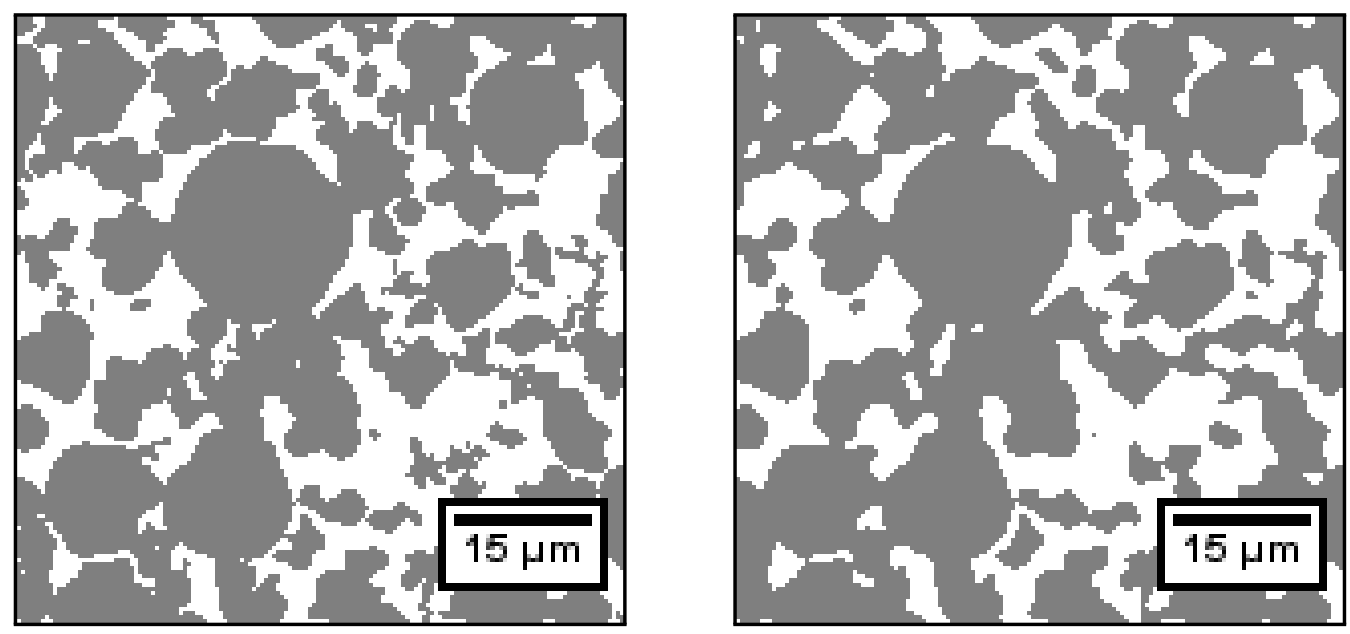

Figure 3: Two-dimensional in-plane cutout $(65.7 \mu \mathrm{m} \times 65.7 \mu \mathrm{m})$ of virtually generated cathode compacted with $500 \mathrm{MPa}$, before (left) and after (right) the post-processing step.

\subsection{Correction factor}

The last model parameter that has to be computed is the correction factor $\rho>0$. This parameter plays a major role in the creation of particles via spherical harmonics. The expected volume of the $i$-th particle is given by the volume of a ball with radius $\rho R_{i}$, where $R_{i}$ denotes the radius of the underlying sphere. The correction factor comes into play, since the initially used packing density $\xi$ is not exactly achieved for three reasons. The first reason is that using a coreshell ratio $\rho<1$ leads to a slightly overlapping system of spheres. The second one is that the volume of a discretized spherical harmonics particle is not necessarily equal to the volume of the 20 underlying sphere due to discretization errors. The third one is that the final post processing step can change the volume fraction of the particle phase. In order to compensate for these deviations, we use interpolation search in order to compute the correction factor. More precisely, we generated ten model realizations in each step on the sampling window $W=[0,500] \times[0,500] \times[0, z]$, where the $z$ vales are given in Table 1 , and computed the average volume fraction. The results can be

found in Table 2. 


\section{Model validation}

After having calibrated the stochastic microstructure model to eight different data sets, we will now quantify the goodness of fit. For this purpose, we generate ten model realizations for each compaction load, using the model parameters described in Section 4. For them, we calculate

5 several microstructural characteristics, which we compare with the corresponding characteristics estimated from tomographic image data. All results presented in this section are averaged over ten model realizations or over twelve tomography cutouts, respectively. Figure 4 allows for a first visual comparison between a cutout from the tomography data and a simulated cathode microstructure.

\subsection{Phase-based image characteristics}

To begin with, we consider the volume fraction of NCM-particles, which is shown in the left plot of Figure 5. One can observe that there is an almost perfect match between tomography and simulation, which is not surprising since the correction factor has been computed in such a way that the correct volume fraction is matched.

15

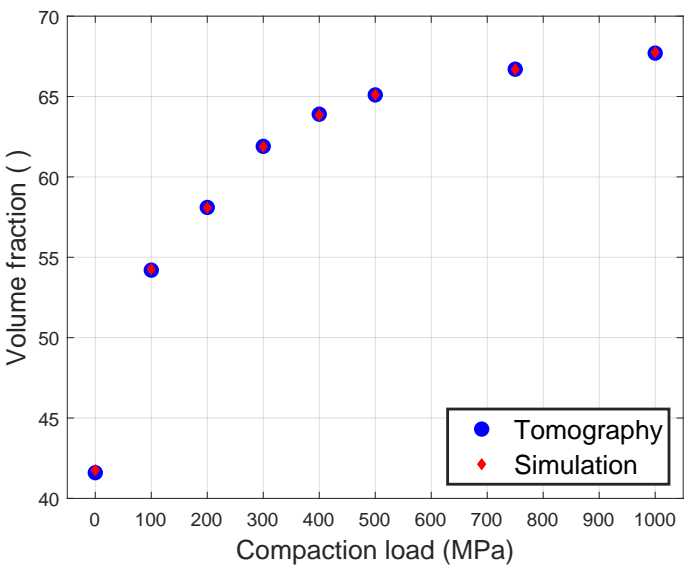

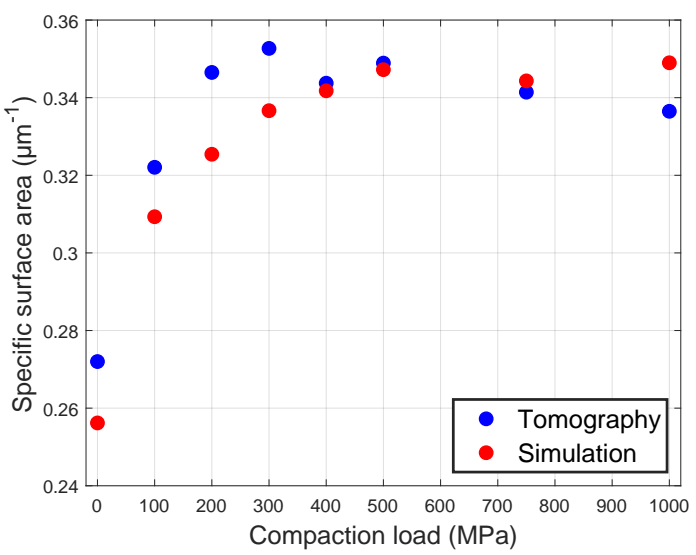

Figure 5: Comparison of volume fraction of active material (left) and its specific surface area (right) for tomography (blue) and the virtually generated microstructures (red).

As it has been shown in [31], geodesic tortuosity essentially influences transport phenomena in porous materials, such as the electrodes of batteries and fuel cells. The distribution of geodesic tortuosity is estimated from image data as described in [15]. The results are shown in Figure 6. It can be observed that the distribution of local geodesic tortuosity is narrower for the simulated 

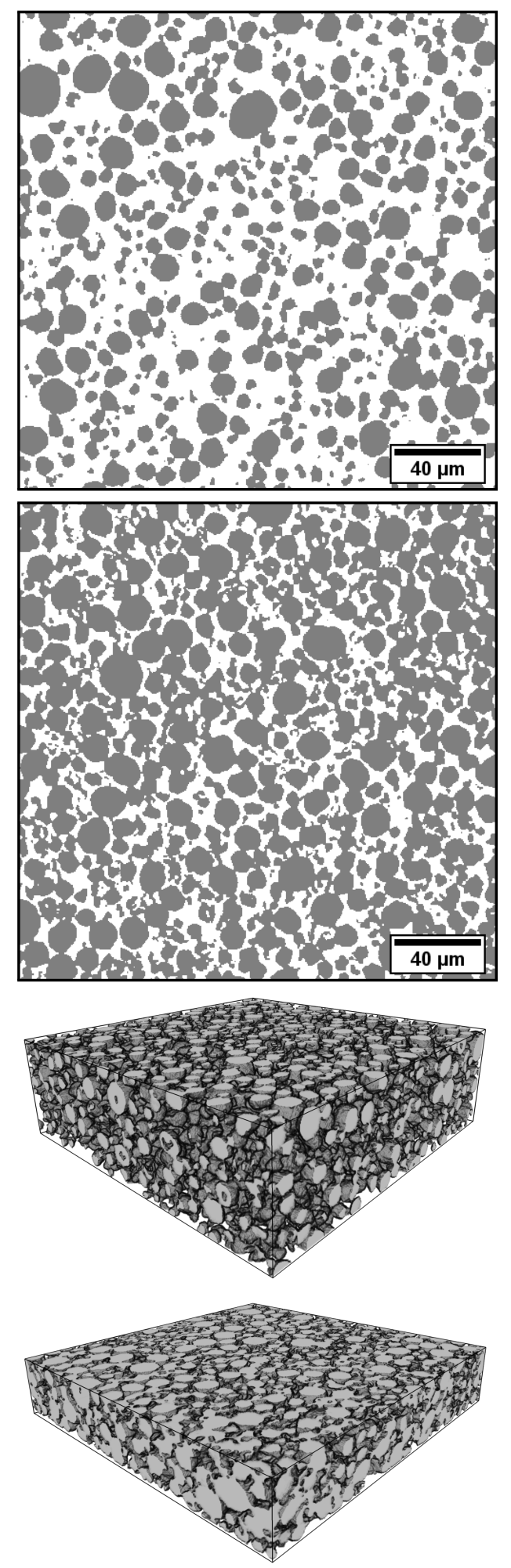
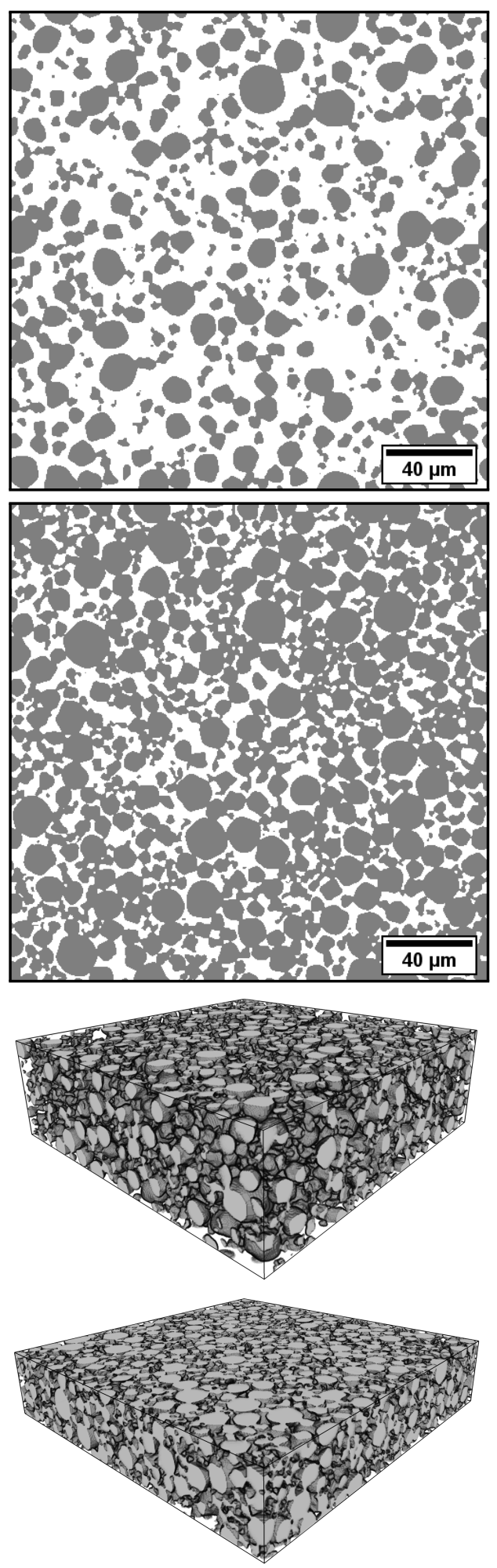

Figure 4: 2D in-plane cutouts $(219 \mu \mathrm{m} \times 219 \mu \mathrm{m})$ and 3D renderings of tomographic image data (left) and virtually generated microstructures (right) for $0 \mathrm{MPa}$ (top) and $750 \mathrm{MPa}$ (bottom). 
microstructures. Nevertheless, the calibrated stochastic model is able to represent the fact that the mean geodesic tortuosity is monotonously increasing for increasing compaction loads. However, it turns out that the mean geodesic tortuosity is slightly underestimated by the stochastic microstructure model, see Figure 7.
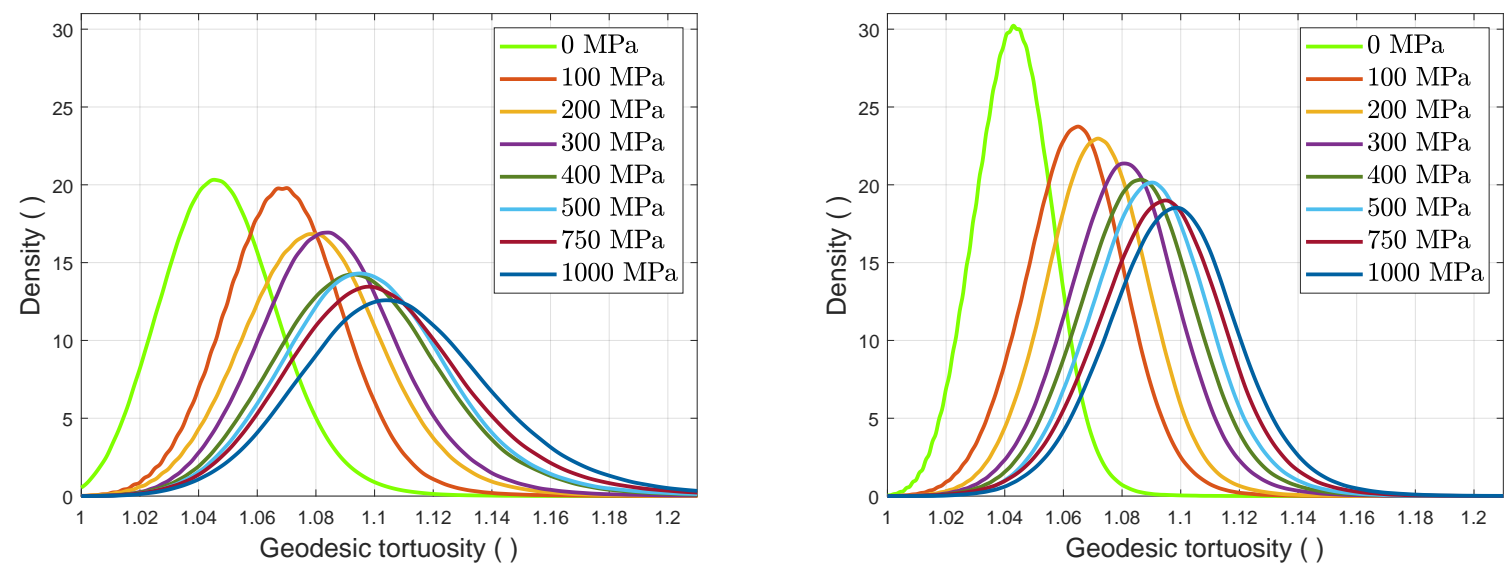

Figure 6: Distribution of local geodesic tortuosity of the bap-phase based on tomography data (left) and model realizations (right).

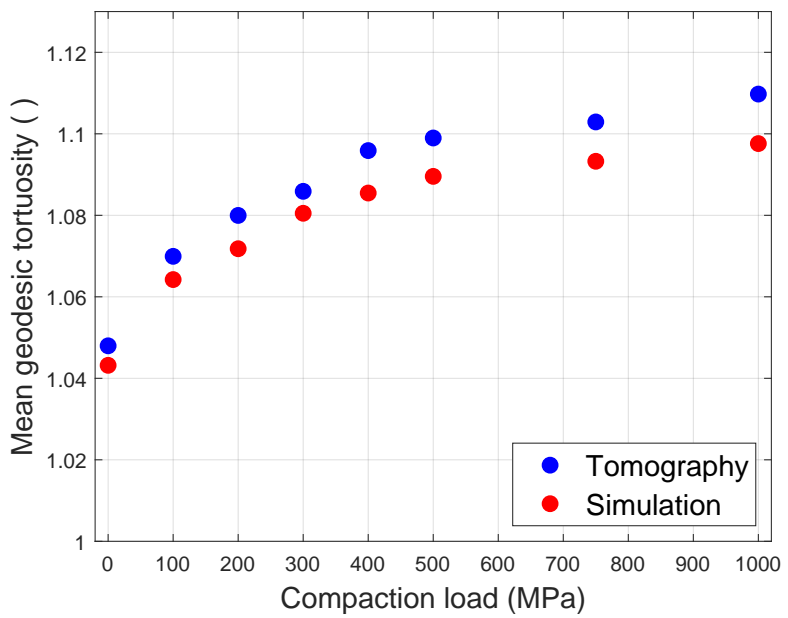

Figure 7: Comparison of mean geodesic tortuosity of the bap-phase for tomography (blue) and the virtually generated microstructures (red).

Another widely used quantity is the continuous phase size distribution (CPSD), which characterizes the morphology of the considered phase, see [32, 33]. Note that for tomographic image data the evolution of the CPSD with increasing compaction load has been analyzed already in [15], where the spatial distribution of binder and conductive additives has been modeled in order to consider the "real" pore space. In the present paper, we calculate the CPSD with respect to the bap-phase, since we do not explicitly distinguish between binder, conductive additives and pore space. Note that we consider the normalized CPSD for better comparability, i.e., we divide the CPSD by the volume of the bap-phase. Figure 8 shows that the stochastic model nicely reflects the behavior of this characteristic. 

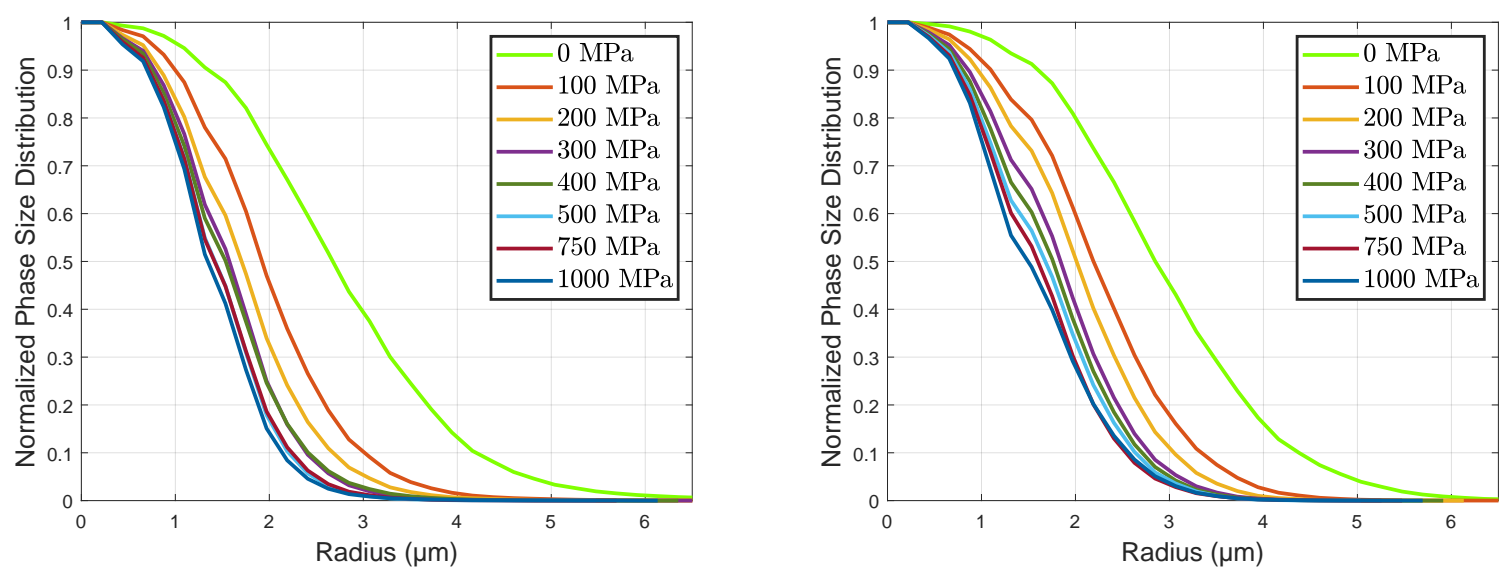

Figure 8: Normalized continuous phase size distribution of the bap-phase based on tomography data (left) and model realizations (right).

Next, we discuss the results of simulated mercury intrusion porosimetry (MIP), see [32]. This direction-dependent characteristic is computed in through-plane direction from "separator" to "current collector" and allows to characterize the strength of bottlenecks. Analogously to the CPSD considered above, we normalize this characteristic. Figure 9 shows a high accordance between tomography and simulation. Furthermore, both plots indicate that (especially for higher compaction loads) there is a characteristics bottleneck radius, i.e., a radius at which a significant decrease of the MIP curve can be observed.
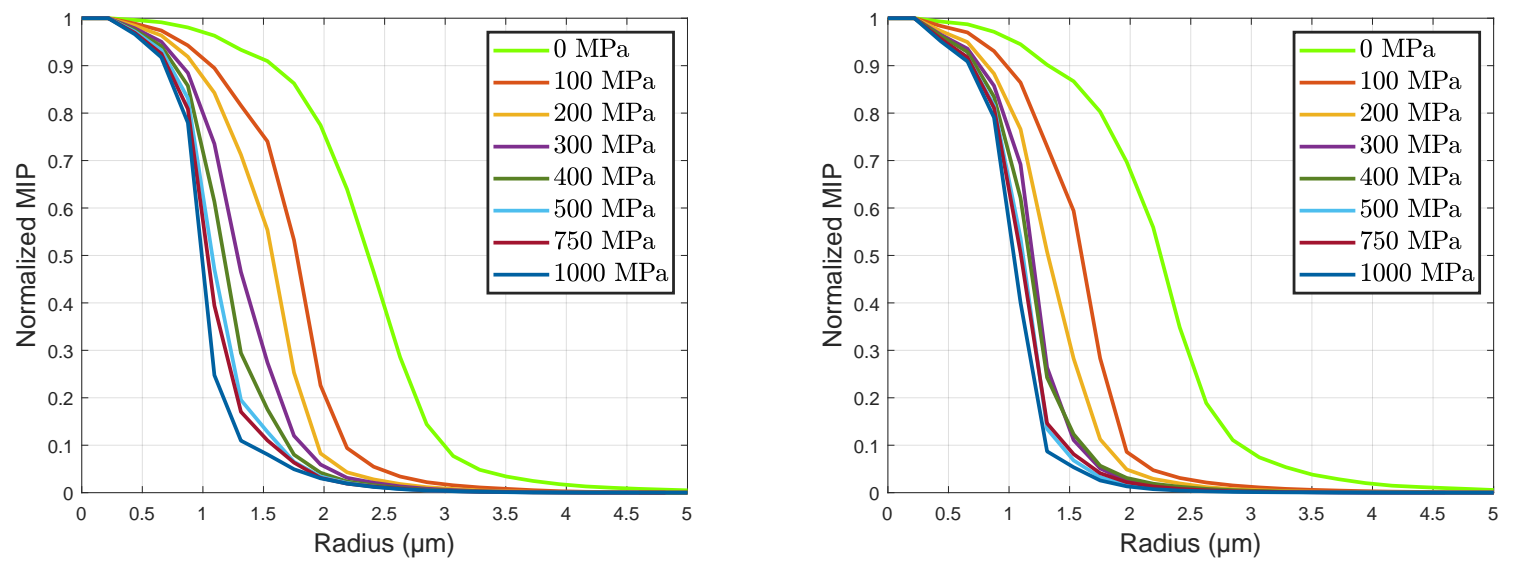

Figure 9: Results for (normalized) simulated mercury intrusion porosimetry of the bap-phase based on tomography data (left) and model realizations (right).

Finally, we investigate the constrictivity of the bap-phase. Note that the constrictivity $\beta$ is defined as $\beta=\left(\frac{r_{\min }}{r_{\max }}\right)^{2} \in[0,1]$, where $r_{\min }$ and $r_{\max }$ are given by the radii for which the normalized MIP and the normalized CPSD take the value 0.5, respectively [33]. Values of $\beta$ close to one indicate that there are almost no constrictions, whereas the presence of strong bottlenecks is indicated in the case when $\beta$ is close to zero. As it can be seen in Figure 10, there is only a minor deviation between tomography and simulation with regard to $r_{\min }$. On the other hand, the stochastic microstructure model tends to slightly overestimate $r_{\max }$, which leads to an underestimation of constrictivity. The comparatively large relative errors regarding constrictivity for higher 
compaction loads are caused by the definition of $\beta$. However, the monotonous behavior of $r_{\text {min }}$, $r_{\max }$ as well as $\beta$ for increasing compaction load is nicely reflected by the calibrated model.

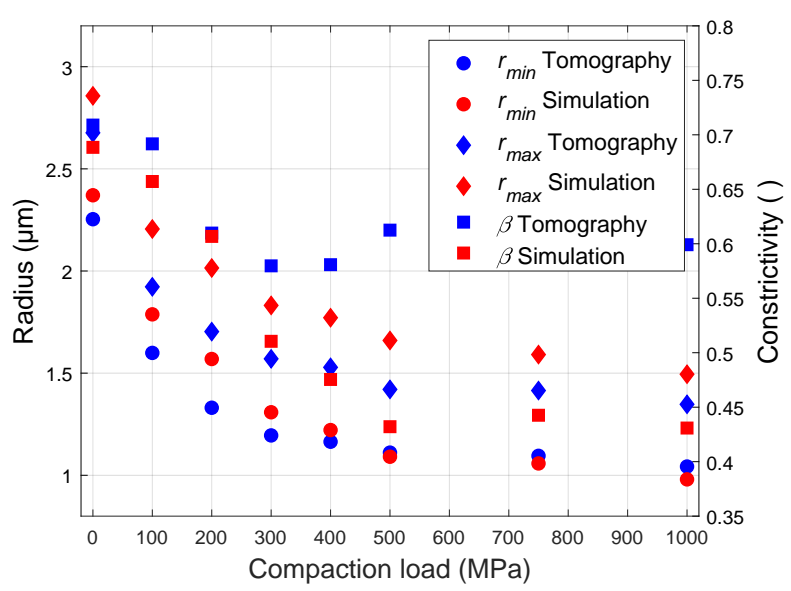

Figure 10: Comparison of $r_{\min }, r_{\max }$ and $\beta$ of the bap-phase for tomography (blue) and the virtually generated microstructures (red).

\subsection{Particle-based image characteristics}

After having focused on phase-based characteristics, we now concentrate on particle-based characteristics. The particle-based segmentation of the virtual microstructures can be easily computed since the analytical description of each particle is known. Hence, each center of mass serves as a marker for a marker-based watershed algorithm [34]. The image used for the flooding process is given by the negative Euclidean distance transform of the binary image.

At first, we consider the sphericity of the NCM-particles. This quantity has been introduced by Wadell in 1932 in order to measure the roundness of rock particles [35]. For a particle with surface area $S$ and volume $V$, the sphericity $\psi \in[0,1]$ is defined as

$$
\psi=\frac{\sqrt[3]{\pi}}{S}(6 V)^{\frac{2}{3}}
$$

Figure 11 provides a comparison of the sphericity distribution of particles obtained from tomographic image data and model realizations. The shape of the distribution is nicely resembled for 0 and $100 \mathrm{MPa}$, whereas the shift of the sphericity distribution to the left observed in tomographic image data for higher compaction loads is not captured by the stochastic microstructure model. This might be caused by the fact that the contact area between active material particles in the tomographic image data is larger than for the simulated microstructures, which leads to more pronounced watershed lines that potentially decrease the sphericity. Nevertheless, both tomography as well as simulation exhibit almost spherically shaped particles. 

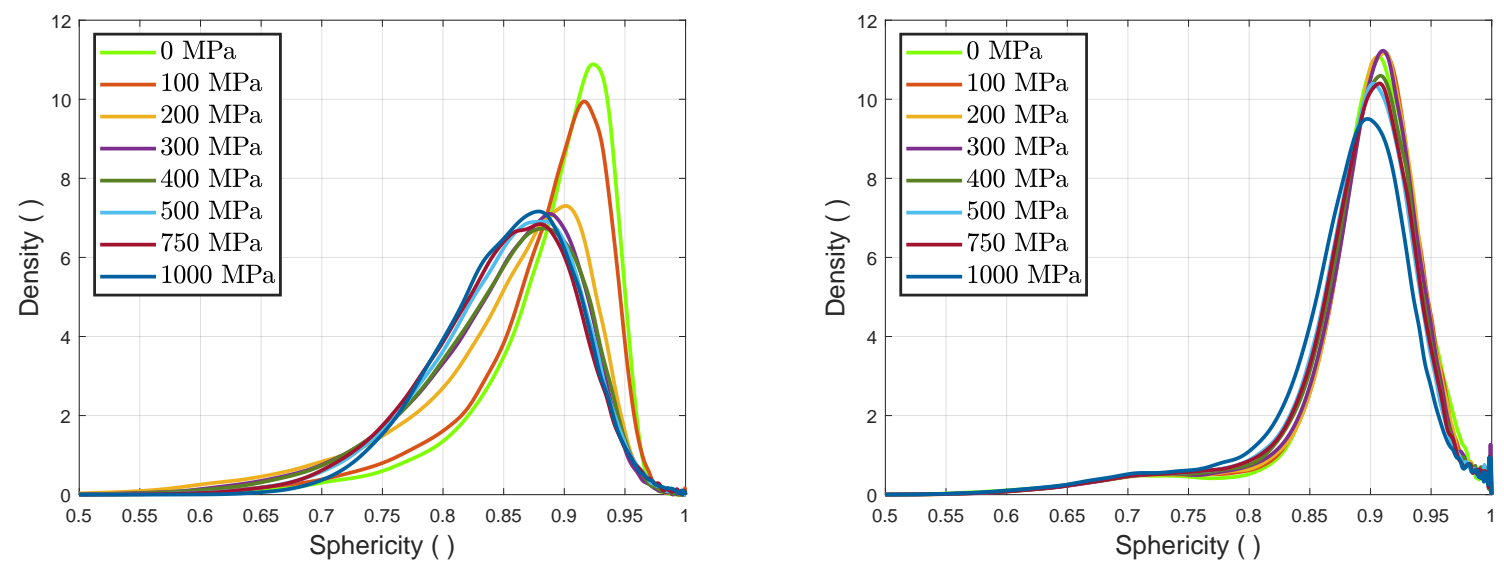

Figure 11: Distribution of sphericity of NCM-particles based on tomography data (left) and simulation (right).

Another particle-based characteristic, which has been investigated in [15] is the particle size distribution, where we average over all compaction loads since the particle size distribution does not depend on the compaction load. Figure 12, left, shows that the particle sizes are slightly smaller for images drawn from the stochastic microstructure model compared to those in the tomographic 5 images. In particular, the virtually generated cathodes contain particles with a radius smaller than the lower bound $\hat{s}=0.503 \mu \mathrm{m}$. This effect comes from the discretization of the particles on a voxel grid. Furthermore, the particles may overlap slightly, but the overlapping part is evenly assigned to both particles in the watershed algorithm. To show this, we consider the sizes of each particle, computed not from the voxel grid, but directly from its analytical representation. The blue curve in Figure 12 clearly shows that in this case the particle size distribution is in high accordance to the predetermined shifted and truncated Birnbaum-Saunders distribution (red curve).

Last but not least, we consider the mean connectivity number, i.e. the mean number of particles that touch a given particle. The results obtained for this quantity are visualized in Figure 12, right, which exhibits an almost perfect coincidence of the results for between tomography and simulation, which is attributed to the computation of the distance threshold $d$ via the bisection method.

To briefly summarize this section, we obtained a high accordance between tomography and simulation for several characteristics, as for example volume fraction of active material, mean geodesic tortuosity of the bap-phase and mean connectivity number. With regard to other quantities, slight deviations occur which is attributed to the fact that our goal is not to perfectly match all characteristics but to develop a parametric stochastic microstructure model, which allows for interpolation between the compaction loads, see Section 6 .

\section{Prediction and interpolation}

This section combines the result stated in the previous sections with regression analysis to predict the model parameters and hence the morphology for an arbitrary compaction load between $0 \mathrm{MPa}$ and $1000 \mathrm{MPa}$. In the following, we only consider model parameters, which depend on the compaction load, whereas the particle size distribution, the angular power spectrum and the series expansion parameter $L$ are chosen as described in Section 4 . 

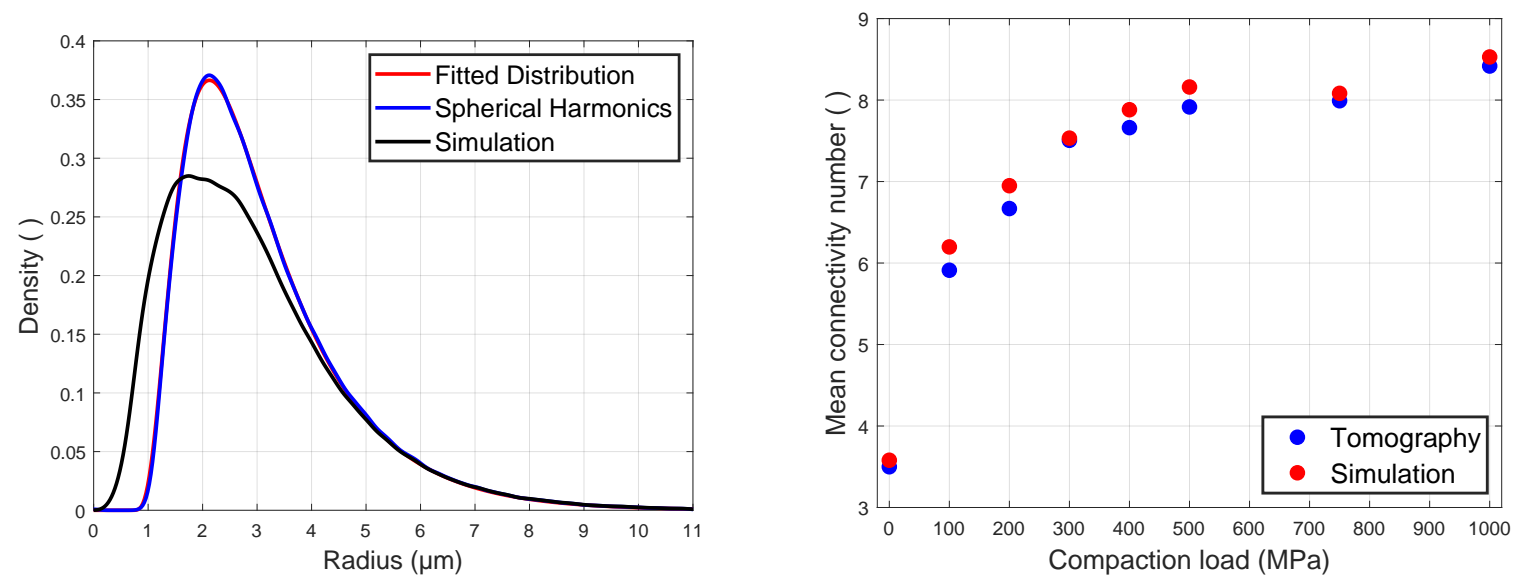

Figure 12: Left: Kernel density estimation of particle size distribution using volume-equivalent radii based on the theoretical volume of spherical harmonics particles (blue), the simulated microstructures including the postprocessing step (black) and the fitted Birnbaum-Saunders distribution (red). Right: Comparison of mean coordination number for tomography (blue) and the simulated microstructures (red).

\subsection{Interpolation between experimentally analyzed compaction loads}

In this section, we present suitable analytic functions for fitting the four model parameters that depend on the compaction load. To begin with, we consider the volume fraction of NCM-particles, which is preset in the first modeling step by the volume fraction of the sphere packing. In order to predict this quantity for arbitrary compaction loads, we use the rational function given by

$$
f(x)=\frac{a x+b}{x+c} \text { for all } x \geq 0
$$

Using the parameters for the eight scenarios to which the model has been fitted in Section 4, by least-squares regression, we obtain $\hat{a}=0.7171, \hat{b}=61.51$ and $\hat{c}=147.7$. The predicted results compared to the volume fractions observed in tomographic image data are shown in Figure 13.

In addition, we predict the mean cathode thickness for arbitrary compaction loads using the rational function given in Equation (4) again, which leads to the following values: $\hat{a}=46.5068, \hat{b}=$ 6950.8 and $\hat{c}=82.6356$. Figure 13 shows a nearly perfect fit for this model parameter.

The third parameter is the distance threshold $d$ for the construction of the connectivity graph. Once again, it turns out that the rational function given in Equation (4) is a meaningful choice, which in this case leads to $\hat{a}=2.931, \hat{b}=745.7$ and $\hat{c}=632.8$, see Figure 13.

Since the angular power spectrum does not depend on the compaction load, the creation of particles involves only one model parameter that depends on the compaction load, i.e., the correction factor $\rho$. For this regression problem, a polynomial of second degree provides a good fit, see Figure 13. More precisely, we obtain the regression parameters $\hat{p}_{2}=-1.402 \cdot 10^{-8}, \hat{p}_{1}=2.175 \cdot 10^{-5}$ and $\hat{p}_{0}=1.004$ for the regression model given by

$$
g(x)=p_{2} x^{2}+p_{1} x+p_{0} \text { for all } x \geq 0 .
$$



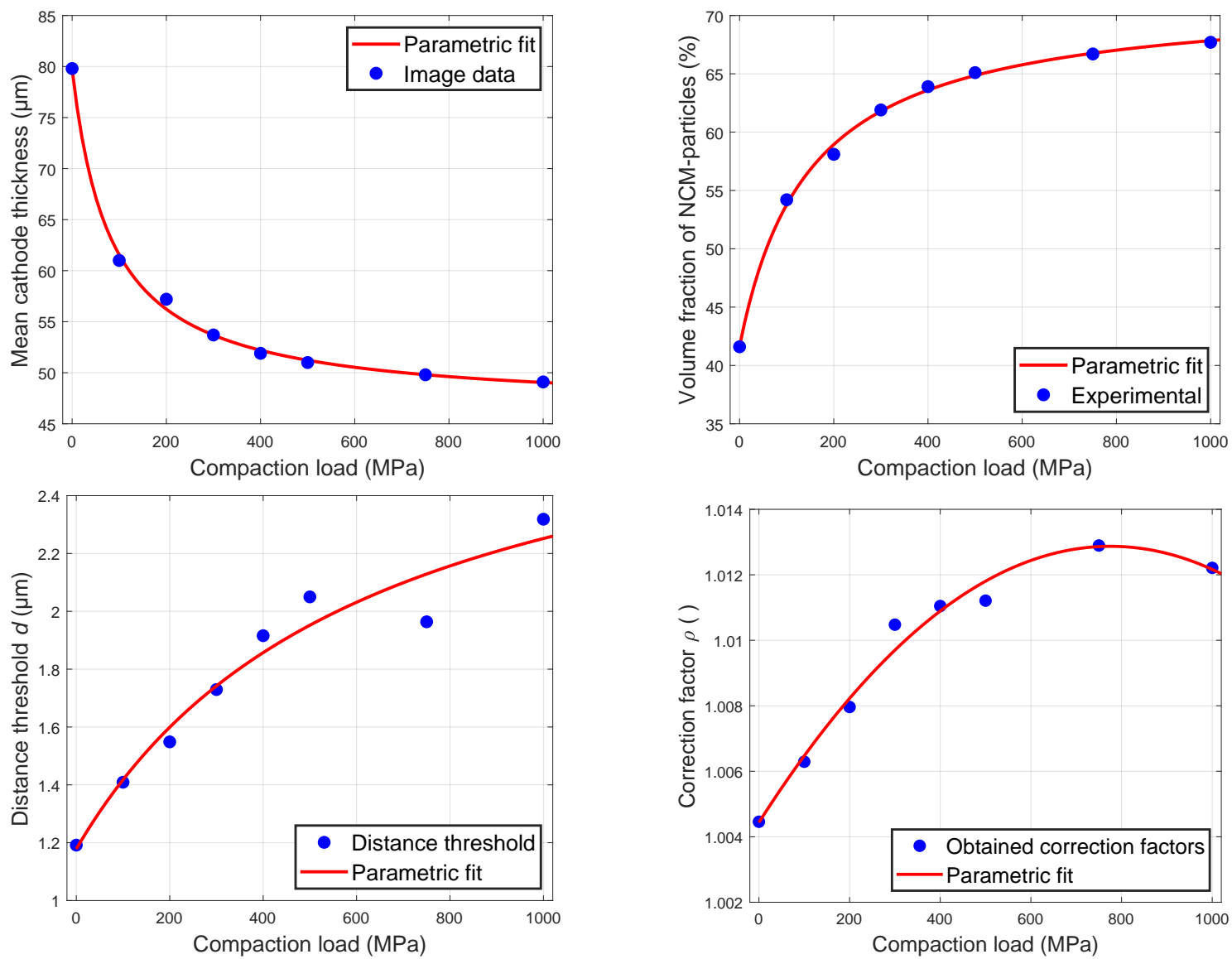

Figure 13: Prediction of mean cathode thickness (top left), volume fraction of NCM-particles (top right), distance threshold $d$ (bottom left) and correction factor $\rho$ (bottom right) by fitting the functions given in Equations (4) and (5), respectively.

\subsection{Leave-one-out cross-validation}

Remember that the regression analysis described above allows us to predict the volume fraction of NCM-particles, the mean cathode thickness, the distance threshold as well as the correction factor for arbitrary compaction loads. Hence, we are able to generate virtual, but realistic microstructures of cathodes for arbitrary compaction loads. In order to validate this approach, we use leave-one-out cross-validation (LOOCV), see [36], i.e., for model fitting we do not use any information concerning a certain experimentally analyzed compaction load, and predict all model parameters based on the remaining seven compaction loads. The resulting simulated microstructures can then be compared to the tomographic image data, which has not been used for the fitting procedure. In the following, we will only present the results for two different compaction loads exemplarily. Additional results for the other compaction loads can be found in the supplementary information.

In order to verify that the previously presented model allows to interpolate between compaction loads, we will leave out the $400 \mathrm{MPa}$ sample for model fitting and compare morphological characteristics of the resulting microstructures with those of tomographic image data as well as model realizations of the calibrated model as described in Section 4. Figure 14 shows that there is nearly 
no difference between the cathode morphologies generated by the calibrated microstructure model and the simulated microstructures generated by using the cross-validation model parameters.

By leaving out the $1000 \mathrm{MPa}$ case for model fitting we can check if the model allows for extrapolation. Both plots in Figure 14 show an almost perfect match between the calibrated model and 5 the model which uses the cross-validation model parameters. This indicates that extrapolation leads to meaningful results. Hence, it can be concluded that the presented model is capable of generating a broad range of virtual, but still realistic cathode morphologies. It should be mentioned that the extrapolation is limited to compaction loads that do not cause fundamental changes of the compaction mechanisms. In the supplementary information, several scalar microstructure characteristics are given, where each of the eight compaction loads has been left out for model fitting.
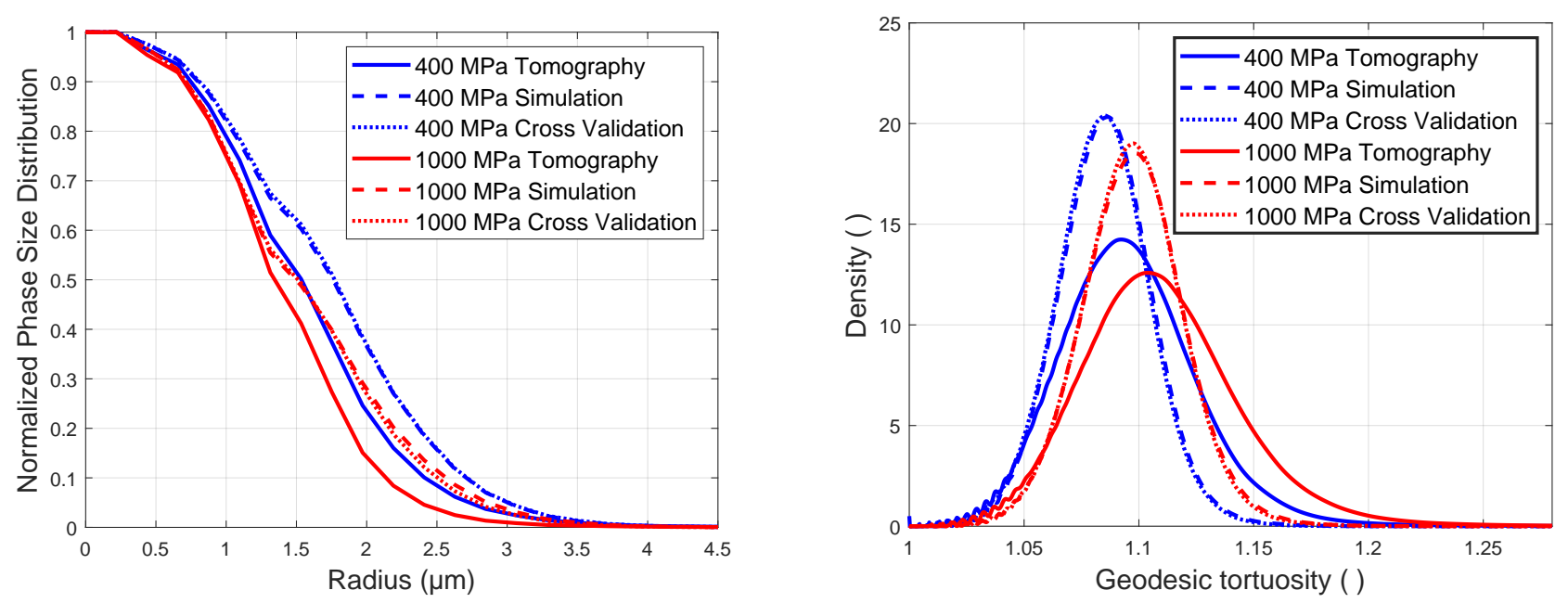

Figure 14: Continuous phase size distribution (left) and the distribution of local geodesic tortuosity (right) for $400 \mathrm{MPa}$ (blue) and $1000 \mathrm{MPa}$ (red). Both characteristics have been computed for the bap-phase.

\subsection{Prediction of microstructure for arbitrary compaction loads}

Since we obtained parametric fits for all compaction load dependent quantities in Sections 6.1 and 6.2, we are now able to predict the model parameters for arbitrary compaction loads. In particular, we present virtually generated microstructures for compaction loads of $50 \mathrm{MPa}, 600 \mathrm{MPa}$ and $1100 \mathrm{MPa}$, respectively. The cutouts of virtually generated microstructures shown in Figure 15 seem to be plausible. In order to characterize these microstructures more formally, we generated ten model realizations for each compaction load and computed various morphological characteristics.

The averaged values obtained for them are summarized in Table 3. In particular, Table 3 contains the predicted compaction-load-dependent model parameters. 

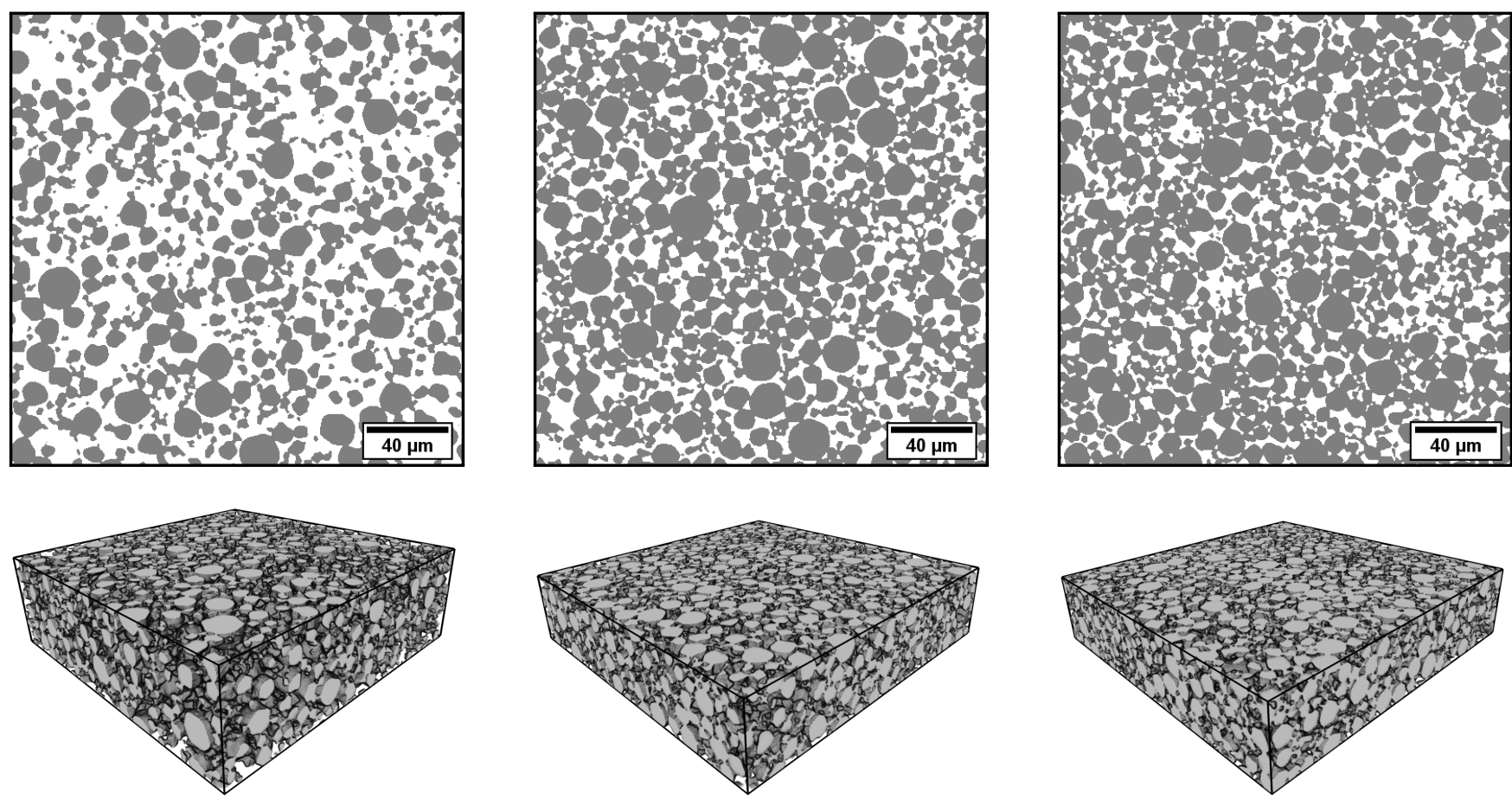

Figure 15: Virtually generated microstructures for $50 \mathrm{MPa}$ (left), $600 \mathrm{MPa}$ (center) and $1100 \mathrm{MPa}$ (right). Top: Two-dimensional in-plane cutouts $(219 \mu \mathrm{m} \times 219 \mu \mathrm{m})$. Bottom: Three-dimensional visualization of simulated microstructures.

Table 3: Model parameters (first four rows), together with averaged characteristics of virtually generated microstructures for compaction loads of $50 \mathrm{MPa}, 600 \mathrm{MPa}$ and $1100 \mathrm{MPa}$.

\begin{tabular}{lccc}
\hline Compaction load $(\mathrm{MPa})$ & 50 & 600 & 1100 \\
\hline Correction factor $\rho()$ & 1.005 & 1.012 & 1.011 \\
Size in $z$-direction () & 152 & 114 & 111 \\
Packing density $\xi(\%)$ & 49.2 & 65.8 & 68.2 \\
Distance threshold $d(\mu \mathrm{m})$ & 1.306 & 2.031 & 2.291 \\
Volume fraction $(\%)$ & 49.3 & 65.8 & 67.9 \\
Specific surface area $\left(\mathrm{\mu m}^{-1}\right)$ & 0.291 & 0.345 & 0.350 \\
Mean connectivity number ( ) & 5.25 & 8.15 & 8.53 \\
Mean geodesic tortuosity ( ) & 1.055 & 1.09 & 1.10 \\
\hline
\end{tabular}

\section{Conclusion}

In this paper, we calibrated one and the same parametric microstructure model type to eight differently compacted cathodes. The virtually generated cathodes nicely reflect the morphological properties of the 3D tomographic image data, like, for example, the volume fraction of the active 5 material, its specific surface area, the geodesic tortuosity of the bap-phase and the connectedness of particles. In addition, all model parameters can be predicted for arbitrary compaction loads. This allows us to generate a broad range of virtual, but realistic cathode morphologies for arbitrary 
compaction loads, reducing the cost of manufacturing and imaging. The simulated microstructures can be used in numerical simulation of electrochemical properties in order to better understand and quantify the influence of the cathode morphology on cell performance.

\section{Data availability}

The raw/processed data required to reproduce these findings cannot be shared at this time as the data also forms part of an ongoing study.

\section{References}

[1] B. Scrosati, K. M. Abraham, W. van Schalkwijk, and J. Hassoun, eds., Lithium Batteries: Advanced Technologies and Applications. The Electrochemical Society Series, Hoboken, NJ: Wiley, 2013.

[2] W. Li and J. C. Currie, "Morphology effects on the electrochemical performance of $\mathrm{LiNi}_{1-x} \mathrm{Co}_{x} \mathrm{O}_{2}$," Journal of The Electrochemical Society, vol. 144, no. 8, p. 2773-2779, 1997.

[3] Y. Shin and A. Manthiram, "Influence of microstructure on the electrochemical performance of $\mathrm{LiMn}_{2-y-z} \mathrm{Li}_{y} \mathrm{Ni}_{z} \mathrm{O}_{4}$ spinel cathodes in rechargeable lithium batteries," Journal of Power Sources, vol. 126, no. 1, p. $169-174,2004$.

[4] S. Cho, C.-F. Chen, and P. P. Mukherjee, "Influence of microstructure on impedance response in intercalation electrodes," Journal of The Electrochemical Society, vol. 162, no. 7, p. A1202A1214, 2015.

[5] J. Wilson, J. Cronin, S. Barnett, and S. Harris, "Measurement of three-dimensional microstructure in a $\mathrm{LiCoO}_{2}$ positive electrode," Journal of Power Sources, vol. 196, no. 7, p. 3443-3447, 2011.

[6] A. H. Wiedemann, G. M. Goldin, S. A. Barnett, H. Zhu, and R. J. Kee, "Effects of threedimensional cathode microstructure on the performance of lithium-ion battery cathodes," Electrochemica Acta, vol. 88, p. 580-588, 2013.

[7] Z. Wang, L. Zhou, and X. W. (David) Lou, "Metal oxide hollow nanostructures for lithium-ion batteries," Advanced Materials, vol. 24, no. 14, p. 1903-1911, 2012.

[8] A. Vu, Y. Qian, and A. Stein, "Porous electrode materials for lithium-ion batteries - how to prepare them and what makes them special," Advanced Energy Materials, vol. 2, no. 9, p. 1056-1085, 2012.

[9] W. Haselrieder, S. Ivanov, D. K. Christen, H. Bockholt, and A. Kwade, "Impact of the calendering process on the interfacial structure and the related electrochemical performance of secondary lithium-ion batteries," ESC Transactions, vol. 50, no. 26, p. 59-70, 2013.

[10] H. Zheng, L. Tan, G. Liu, X. Song, and V. S. Battaglia, "Calendering effects on the physical and electrochemical properties of $\mathrm{Li}\left[\mathrm{Ni}_{1 / 3} \mathrm{Mn}_{1 / 3} \mathrm{Co}_{1 / 3}\right] \mathrm{O}_{2}$ cathode," Journal of Power Sources, vol. 208, p. 52-57, 2012. 
[11] J. Gnanaraj, Y. S. Cohen, M. Levi, and D. Aurbach, "The effect of pressure on the electroanalytical response of graphite anodes and $\mathrm{LiCoO}_{2}$ cathodes for Li-ion batteries," Journal of Electroanalytical Chemistry, vol. 516, no. 1, p. 89-102, 2001.

[12] G. Lenze, F. Röder, H. Bockholt, W. Haselrieder, A. Kwade, and U. Krewer, "Simulationsupported analysis of calendering impacts on the performance of lithium-ion-batteries," Journal of The Electrochemical Society, vol. 164, no. 6, p. A1223-A1233, 2017.

[13] H. Bockholt, M. Indrikova, A. Netz, F. Golks, and A. Kwade, "The interaction of consecutive process steps in the manufacturing of lithium-ion battery electrodes with regard to structural and electrochemical properties," Journal of Power Sources, vol. 325, p. 140-151, 2016.

[14] G. Lenze, H. Bockholt, C. Schilcher, L. Froböse, D. Jansen, U. Krewer, and A. Kwade, "Impacts of variations in manufacturing parameters on performance of lithium-ion batteries," Journal of The Electrochemical Society, vol. 165, no. 2, p. A314-A322, 2018.

[15] K. Kuchler, B. Prifling, D. Schmidt, H. Markötter, I. Manke, T. Bernthaler, V. Knoblauch, and V. Schmidt, "Analysis of the 3D microstructure of experimental cathode films for lithiumion batteries under increasing compaction," Journal of Microscopy, vol. 272, no. 2, p. 96-110, 2018.

[16] S. N. Chiu, D. Stoyan, W. S. Kendall, and J. Mecke, Stochastic Geometry and its Applications. Chichester: J. Wiley \& Sons, $3^{\text {rd }}$ ed., 2013.

[17] D. Westhoff, J. Skibinski, O. Šedivý, B. Wysocki, T. Wejrzanowski, and V. Schmidt, "Investigation of the relationship between morphology and permeability for open-cell foams using virtual materials testing," Materials \& Design, vol. 147, p. 1-10, 2018.

[18] G. Gaiselmann, M. Neumann, V. Schmidt, O. Pecho, T. Hocker, and L. Holzer, "Quantitative relationships between microstructure and effective transport properties based on virtual materials testing," AIChE Journal, vol. 60, no. 6, p. 1983-1999, 2014.

[19] M. Neumann, O. Stenzel, F. Willot, L. Holzer, and V. Schmidt, "Quantifying the influence of microstructure on effective conductivity and permeability: virtual materials testing," International Journal of Solids and Structures, 2019. Submitted.

[20] D. Schmidt, M. Kamlah, and V. Knoblauch, "Highly densified NCM-cathodes for high energy Li-ion batteries: Microstructural evolution during densification and its influence on the performance of the electrodes," Journal of Energy Storage, vol. 17, p. 213 - 223, 2018.

[21] D. Westhoff, I. Manke, and V. Schmidt, "Generation of virtual lithium-ion battery electrode microstructures based on spatial stochastic modeling," Computational Materials Science, vol. 151, p. 53-64, 2018.

[22] J. Mosćiński, M. Bargieł, Z. Rycerz, and P. Jakobs, "The force-biased algorithm for the irregular close packing of equal hard spheres," Molecular Simulation, vol. 3, p. 201-212, 1989.

[23] A. Bezrukov, M. Bargieł, and D. Stoyan, "Statistical analysis of simulated random packings of spheres," Particle \& Particle Systems Characterization, vol. 19, no. 2, p. 111-118, 2002. 
[24] J. Feinauer, A. Spettl, I. Manke, S. Strege, A. Kwade, A. Pott, and V. Schmidt, "Structural characterization of particle systems using spherical harmonics," Materials Characterization, vol. 106, p. 123-133, 2015.

[25] Z. Birnbaum and S. Saunders, "A new family of life distributions," Journal of Applied Probability, vol. 6, no. 2, p. 319-327, 1969.

[26] D. Kincaid, D. Kincaid, and E. Cheney, Numerical Analysis: Mathematics of Scientific Computing. Pure and applied undergraduate texts, American Mathematical Society, 2009.

[27] K. Kuchler, D. Westhoff, J. Feinauer, T. Mitsch, I. Manke, and V. Schmidt, "Stochastic model for the 3D microstructure of pristine and cyclically aged cathodes in Li-ion batteries," Modelling and Simulation in Materials Science and Engineering, vol. 26, no. 3, p. 035005, 2018.

[28] W. Burger and M. J. Burge, Digital Image Processing: An Algorithmic Introduction Using Java. Texts in Computer Science, New York: Springer, $1^{\text {st }}$ ed., 2010.

[29] N. Otsu, "A threshold selection method from gray-level histograms," IEEE Transactions on Systems, Man and Cybernetics, vol. 9, no. 1, p. 62-66, 1979.

[30] K. Schladitz, J. Ohser, and W. Nagel, "Measuring intrinsic volumes in digital 3D images," in 13th International Conference Discrete Geometry for Computer Imagery (A. Kuba, L. Nyúl, and K. Palágyi, eds.), p. 247-258, Springer, 2007.

[31] O. Stenzel, O. M. Pecho, L. Holzer, M. Neumann, and V. Schmidt, "Predicting effective conductivities based on geometric microstructure characteristics," AIChE Journal, vol. 62, no. 5, p. 1834-1843, 2016.

[32] B. Münch and L. Holzer, "Contradicting geometrical concepts in pore size analysis attained with electron microscopy and mercury intrusion," Journal of the American Ceramic Society, vol. 91, no. 12, p. 4059-4067, 2008.

[33] L. Holzer, D. Wiedenmann, B. Münch, L. Keller, M. Prestat, P. Gasser, I. Robertson, and B. Grobéty, "The influence of constrictivity on the effective transport properties of porous layers in electrolysis and fuel cells," Journal of Materials Science, vol. 48, p. 2934-2952, 2013.

[34] S. Beucher and F. Meyer, "The morphological approach to segmentation: the watershed transformation," in Mathematical Morphology in Image Processing (E. R. Dougherty, ed.), p. 433481, New York: Marcel Dekker, Inc., 1993.

[35] H. Wadell, "Volume, shape, and roundness of rock particles," The Journal of Geology, vol. 40, no. 5, p. 443-451, 1932.

[36] T. Hastie, R. Tibshirani, and J. Friedman, The Elements of Statistical Learning. Springer, $2^{\text {nd }}$ ed., 2009. 


\section{Supplementary information}

This supplement contains the cross-validation results for those compaction loads, which have not been discussed in Section 6.2. The first row contains the compaction load, which has been left out during cross-validation. The microstructural characteristics are averaged over ten model 5 realizations.

Table A: Predicted values of the compaction-load-dependent model parameters for different compaction loads.

\begin{tabular}{lcccccccc}
\hline Compaction load $(\mathrm{MPa})$ & 0 & 100 & 200 & 300 & 400 & 500 & 750 & 1000 \\
\hline Correction factor $\rho()$ & 1.0044 & 1.0065 & 1.0083 & 1.0095 & 1.0108 & 1.0121 & 1.0128 & 1.0119 \\
Voxels in z-direction () & 168 & 142 & 128 & 123 & 119 & 117 & 114 & 112 \\
Packing density $\xi(\%)$ & 43.3 & 53.4 & 59.3 & 61.8 & 63.5 & 64.8 & 66.8 & 68.0 \\
Distance threshold $d(\mu \mathrm{m})$ & 1.107 & 1.420 & 1.617 & 1.746 & 1.840 & 1.926 & 2.197 & 2.150 \\
\hline
\end{tabular}

Table B: Averaged microstructure characteristics for different compaction loads, which have been left out during cross-validation.

\begin{tabular}{|c|c|c|c|c|c|c|c|c|}
\hline Compaction load (MPa) & 0 & 100 & 200 & 300 & 400 & 500 & 750 & 1000 \\
\hline Volume fraction $(\%)$ & 43.4 & 53.6 & 59.2 & 61.7 & 63.5 & 64.8 & 66.9 & 67.7 \\
\hline Rel. error w.r.t. tomography (\%) & 4.24 & 1.09 & 1.94 & 0.37 & 0.64 & 0.40 & 0.31 & 0.07 \\
\hline Rel. error w.r.t. simulation (\%) & 0.37 & 0.11 & 0.03 & 0.03 & 0.08 & 0.02 & 0.02 & 0.07 \\
\hline Specific surface area $\left({\left.\mu m^{-1}\right)}^{-1}\right.$ & 0.265 & 0.309 & 0.330 & 0.337 & 0.341 & 0.343 & 0.347 & 0.350 \\
\hline Rel. error w.r.t. tomography (\%) & 2.77 & 4.20 & 4.89 & 4.44 & 0.76 & 1.78 & 1.62 & 3.88 \\
\hline Rel. error w.r.t. simulation (\%) & 5.81 & 3.95 & 6.08 & 4.55 & 0.57 & 0.48 & 0.85 & 3.71 \\
\hline Mean connectivity number ( ) & 3.72 & 6.10 & 7.19 & 7.57 & 7.78 & 7.97 & 8.36 & 8.39 \\
\hline Rel. error w.r.t. tomography (\%) & 6.27 & 3.29 & 7.72 & 0.79 & 1.62 & 0.68 & 4.66 & 0.32 \\
\hline Rel. error w.r.t. simulation (\%) & 2.29 & 4.87 & 4.18 & 0.30 & 2.89 & 3.03 & 1.16 & 1.29 \\
\hline Mean geodesic tortuosity ( ) & 1.046 & 1.063 & 1.074 & 1.080 & 1.085 & 1.088 & 1.095 & 1.097 \\
\hline Rel. error w.r.t. tomography (\%) & 0.24 & 0.67 & 0.53 & 0.56 & 1.04 & 0.97 & 0.74 & 1.14 \\
\hline Rel. error w.r.t. simulation (\%) & 0.46 & 0.53 & 0.75 & 0.50 & 0.95 & 0.86 & 0.88 & 1.09 \\
\hline$r_{\min }(\mu \mathrm{m})$ & 2.24 & 1.80 & 1.49 & 1.30 & 1.19 & 1.13 & 1.01 & 1.00 \\
\hline Rel. error w.r.t. tomography (\%) & 0.52 & 12.46 & 12.26 & 9.07 & 2.52 & 1.77 & 7.61 & 4.38 \\
\hline Rel. error w.r.t. simulation (\%) & 5.20 & 11.80 & 17.93 & 9.48 & 4.90 & 1.81 & 3.40 & 6.02 \\
\hline$r_{\max }(\mu \mathrm{m})$ & 2.72 & 2.22 & 1.96 & 1.85 & 1.77 & 1.70 & 1.58 & 1.51 \\
\hline Rel. error w.r.t. tomography (\%) & 1.51 & 15.62 & 15.19 & 18.07 & 15.93 & 19.96 & 11.34 & 12.23 \\
\hline Rel. error w.r.t simulation (\%) & 6.76 & 14.72 & 18.29 & 16.65 & 15.90 & 16.87 & 12.45 & 10.93 \\
\hline
\end{tabular}

